\title{
Association between mRNA expression of chemotherapy-related genes and clinicopathological features in colorectal cancer: A large-scale population analysis
}

\author{
YUJI SHIMAMOTO ${ }^{1}$, MAMORU NUKATSUKA $^{2}$, TEIJI TAKECHI $^{1}$ and MASAKAZU FUKUSHIMA ${ }^{3}$ \\ ${ }^{1}$ Translational Research Laboratory, Taiho Pharmaceutical Co., Ltd., Uchikanda, Chiyoda-ku, \\ Tokyo 101-0047; ${ }^{2}$ Translational Research Laboratory, Taiho Pharmaceutical Co., Ltd., \\ Kawauchi-Cho, Tokushima-shi, Tokushima 771-0194; ${ }^{3}$ The Japan-Multinational Trial Organization, \\ Naka-ku, Nagoya-shi, Aichi 460-0002, Japan
}

Received July 22, 2015; Accepted November 24, 2015

DOI: $10.3892 / \mathrm{ijmm} .2015 .2427$

\begin{abstract}
To establish the individualized treatment of patients with colorectal cancer, factors associated with chemotherapeutic effects should be identified. However, to the best of our knowledge, few studies are available on this topic, although it is known that the prognosis of patients and sensitivity to chemotherapy depend on the location of the tumor and that the tumor location is important for individualized treatment. In this study, primary tumors obtained from 1,129 patients with colorectal cancer were used to measure the mRNA expression levels of the following genes associated with the effects of standard chemotherapy for colorectal cancer: 5-fluorouracil (5-FU)-related thymidylate synthase (TYMS), dihydropyrimidine dehydrogenase $(D P Y D)$ and thymidine phosphorylase $(T Y M P)$; folate-related dihydrofolate reductase $(D H F R)$, folylpolyglutamate synthase (FPGS) and gamma-glutamyl hydrolase $(G G H)$; irinotecan-related topoisomerase I (TOPI); oxaliplatin-related excision repair cross-complementing 1 (ERCC1); biologic agent-related vascular endothelial growth factor $(V E G F)$ and epidermal growth factor receptor $(E G F R)$. Large-scale population analysis was performed to determine the association of gene expression with the clinicopathological features, in particular, the location of the colorectal cancer. From the results of our analysis of the mRNA expression of these 10 genes, we noted the strongest correlation between DPYD and TYMP, followed by TYMS and DHFR. The location of the colorectal cancer was classified into 4 regions (the right- and left-sided colon, rectosigmoid and rectum) and was compared with gene expres-
\end{abstract}

Correspondence to: Dr Yuji Shimamoto, Translational Research Laboratory, Taiho Pharmaceutical Co., Ltd., 1-2-4 Uchikanda, Chiyoda-ku, Tokyo 101-0047, Japan

E-mail: simamoto@taiho.co.jp

Key words: mRNA expression, chemotherapy-related genes, clinicopathological features, colorectal cancer, population analysis sion. A significant difference in all genes, apart from $V E G F$, was noted. Of the remaining 9 genes, the highest expression of TYMS and DPYD was observed in the right-sided colon; the highest expression of $G G H$ and $E G F R$ was noted in the left-sided colon; the highest expression of DHFR, FPGS, TOPI and $E R C C 1$ was noted in the rectosigmoid, whereas $T Y M P$ expression was approximately equivalent in the right-sided colon and rectum, and higher than that in other locations. The data generated from this study may prove to be useful for the development of individualized chemotherapeutic treatments for patients with colorectal cancer, and will mean that the tumor location is taken into account.

\section{Introduction}

In 2012, colorectal cancer was estimated to be the third most common type of cancer after lung and prostate cancer in men, and the second most common after breast cancer in women worldwide. In terms of the mortality rate, it ranked fourth, after cancer of the lungs, liver and stomach in both men and women (1). Although colorectal cancer is principally treated by surgery, chemotherapy and radiotherapy may be given priority, depending on the stage of the disease. For many years, 5-fluorouracil (5-FU) alone or 5-FU and leucovorin were effectively used as chemotherapeutic agents for the treatment of colorectal cancer. However, the survival benefit in patients with metastatic colorectal cancer has been reported to be markedly increased by the combined use of chemotherapeutic agents, such as irinotecan and oxaliplatin with biological agents, such as bevacizumab for anti-vascular endothelial growth factor (VEGF) antibody therapy and cetuximab for anti-epidermal growth factor receptor (EGFR) antibody therapy, in addition to cytotoxic agents (2).

To increase the effectiveness of chemotherapy for patients with colorectal cancer, it is necessary to establish individualized treatment strategies and, therefore, identifying factors associated with the effects of chemotherapy for colorectal cancer also becomes important. Thymidylate synthase (TYMS), a 5-FU target, requires 5,10-methylenetetrahydrofolate (5,10-methylene-THF), a folate co-factor, as a methyl group donor 
and methylates deoxyuridine monophosphate (dUMP) to become deoxythymidine monophosphate (dTMP). It acts as a rate-limiting enzyme during DNA synthesis (3). As an active metabolite of 5-FU, 5-fluoro-dUMP (FdUMP) covalently links with TYMS and 5,10-methylene-THF, forming a ternary complex and inhibiting the TYMS response (3). Dihydropyrimidine dehydrogenase (DPYD) is a rate-limiting enzyme catalyzing the response during the first step of the catabolism of 5-FU (4). Thymidine phosphorylase (TYMP), which is associated with the metabolism of $5-\mathrm{FU}$, is an enzyme catalyzing the reversible phosphorolysis of 5-FU to 5-fluoro-2'-deoxyuridine. TYMP is identical to platelet-derived endothelial-cell growth factor and is thought to be involved in angiogenesis (5). Salonga et al (6) and Soong et al (7) reported that the mRNA or protein expression of TYMS, DPYD and TYMP was associated with the antitumor effects of 5-FU. Leucovorin is metabolized in vivo into 5,10-methylene-THF, strengthens the ternary complex of FdUMP, TYMS and 5,10-methylene-THF, and strengthens the antitumor effects of 5-FU (8). Dihydrofolate reductase (DHFR) catalyzes the conversion of dihydrofolate into tetrahydrofolate, which is an important step in the generation of 5,10-methylene-THF (3). In addition, folate is converted from the monoglutamate to the polyglutamate form by folylpolyglutamate synthase (FPGS) and can therefore be easily maintained within the cells, while the polyglutamate chain is cleaved by gamma-glutamyl hydrolase (GGH) and is converted into the monoglutamate form. It is thought that the catalyzing of opposite reactions by FPGS and GGH in folate metabolism is associated with the adjustment of folate levels in cells, as well as with the augmentation of the effects of 5-FU by leucovorin (9). Topoisomerase I (TOP1), a target enzyme of irinotecan, destroys the superhelicity observed in DNA during DNA metabolic processes, such as replication and transcription; consequently, TOP1 plays a role in the regulation of DNA topology (10). The association of excision repair cross-complementing 1 (ERCC1), reportedly associated with the effects of oxaliplatin, is an excision nuclease involved in the nucleotide excision repair of DNA and plays an important role in the repair of platinum-induced DNA adducts $(11,12)$. Both TOP1 and ERCC1 maintain the normal structure of DNA and are important for DNA functionality. VEGF is a key mediator in angiogenesis, consisting of multiple steps, and its high-level expression is thought to be closely associated with tumor growth and metastasis (13). EGFR plays an important role in the signaling pathway of epithelial cell growth, and its activation is thought to be closely associated with tumor invasion, metastasis and angiogenesis (14). Both VEGF and EGFR play important roles in signaling pathways associated with cell growth, such as angiogenesis and proliferation.

It is well known that not only embryological, morphological, physiological and molecular features, but also the prognosis of patients and their sensitivity to chemotherapy depend on whether the primary lesion of colorectal cancer is located on the right or left side (15-18). Consequently, the location of the colorectal cancer is thought to be important for deciding individualized treatment for patients with colorectal cancer. However, to the best of our knowledge, to date, there are few studies available on the association between the location of the colorectal cancer and the level of expression of genes related to the effects of chemotherapy for colorectal cancer.
Previously, we performed large-scale population studies investigating TYMS and DPYD activity, as well as protein and mRNA expression in various types of solid tumors and reported basic data regarding the comparisons of assays and the level of expression, according to the carcinoma $(19,20)$. In this study, primary tumors obtained from 1,129 patients with colorectal cancer who received no chemotherapy were used. After measuring the genes related to standard chemotherapy for colorectal cancer (TYMS, DPYD, TYMP, DHFR, FPGS, GGH, $T O P 1, E R C C 1, V E G F$ and $E G F R$ ), a large-scale population analysis was performed for the purpose of determining the association with clinicopathological features, and in particular, the location of the colorectal cancer.

\section{Patients and methods}

Patients and samples. Between January 2008 and June 2012, we collected samples from primary tumor surgeries conducted on 2,017 patients with colorectal cancer in 39 hospitals across Japan. All the hospitals participated in this study after approval was obtained from the ethical review board of each hospital. Formalin-fixed paraffin-embedded (FFPE) tumor specimens were used for the measurement of mRNA expression. The primary lesions of 1,129 patients with colorectal cancer who did not receive any chemotherapy were analyzed for the mRNA expression of 10 target genes (TYMS, DPYD, TYMP, DHFR, FPGS, GGH, TOPI, ERCCI, VEGF and $E G F R$ ). Of these, 888 patients were not included in the analysis: 80 patients $(4.0 \%)$ who received pre-operative chemotherapy; 6 patients $(0.3 \%)$ who were diagnosed with non-colorectal cancer based on a final pathological examination; 630 patients (31.2\%) for whom some of the 10 target genes were not analyzed, at the hospitals' request; 62 patients (3.1\%) for whom none of the 10 target genes were analyzable due to an inadequate number of cancer cells in the FFPE tumor specimens; 110 patients (5.5\%) for whom one or more targeted genes were not analyzable due to insufficient tumor volume or considerable variability in the measured values. In addition, not detectable (n.d.) results were included in the analyzed data. The clinicopathological features of the patients were investigated based on the Japanese classification of colorectal cancer: General Rules for Clinical and Pathological Studies on Cancer of the Colon, Rectum and Anus, 7th edition (21).

Analysis of mRNA expression. The analysis of mRNA expression was performed as previously described (20). Briefly, the tumor sections (10- $\mu \mathrm{m}$-thick) were stained with neutral fast red to enable histological visualization during laser-capture microdissection (PALM Robot-microbeam system; P.A.L.M. Microlaser Technologies AG, Munich, Germany), which was performed to ensure that only the tumor cells were collected. RNA was isolated from the FFPE tumor specimens using a novel proprietary procedure (Response Genetics, Los Angeles, CA, USA; United States Patent No. 6,248,535) and cDNA was obtained as previously described (22). The target cDNA sequences were amplified using quantitative polymerase chain reaction (qPCR) and a fluorescence-based real-time detection method [ABI PRISM 7900 Sequence Detection System (TaqMan); Applied Biosystems, Foster City, CA, USA] as previously described (23-25). The qPCR reaction mixture 
Table I. Clinicopathological features of the 1,129 patients.

No.

Clinicopathological features

of patients $\%$

Age (mean, 66 years; median, 67 years;

range, 24-94 years)

$<67$

$\geq 67$

525

46.5

No available data

558

49.4

Gender

Male

Female

No available data

46

4.1

$662 \quad 58.6$

$459 \quad 40.7$

$\begin{array}{ll}8 & 0.7\end{array}$

Tumor location

Right-sided colon (C, A, T)

324

Left-sided colon (D, S)

317

28.7

Rectosigmoid

117

Rectum

Proctos

No available data

358

4

9

Histology

Well/moderately differentiated

Poorly differentiated

1004

35

Mucinos

Others

No available data

Lymphatic invasion

ly0

ly 1, ly $2,1 y 3$

No available data

91

Venous invasion

v0

v1, v2, v3

No available data

Depth of tumor invasion

M, SM, MP

SS, SE, SI, A, AI

No available data

Lymph node metastasis

N0

$\mathrm{N} 1, \mathrm{~N} 2, \mathrm{~N} 3$

No available data

Stage

0

I

II

III

No available data consisted of primers, dATP, dCTP, dGTP, dUTP, $\mathrm{MgCl}_{2}$ and TaqMan buffer (all reagents were supplied by Applied Biosystems). The qPCR conditions were $50^{\circ} \mathrm{C}$ for $10 \mathrm{sec}$ and $95^{\circ} \mathrm{C}$ for $10 \mathrm{~min}$, followed by 42 cycles at $95^{\circ} \mathrm{C}$ for $15 \mathrm{sec}$ and $60^{\circ} \mathrm{C}$ for $1 \mathrm{~min}$. The $\mathrm{mRNA}$ expression levels were expressed as values relative to those of $\beta$-actin $(A C T B)$, which was used as the internal reference. If the threshold cycle value for a gene of interest was $\geq 39.0$, the expression for that gene was considered to be not detectable (n.d.), but in the case of $A C T B$ it was measured normally.

Statistical analysis. mRNA expression was analyzed as a variable which was converted by using the natural logarithm and producing a normal distribution. Pearson's pairwise correlation coefficient was used to calculate the correlation of intergenic mRNA expression. For hierarchical cluster analysis of mRNA expression, the data were standardized using the means and standard deviation, while Ward's method was used to calculate the distance between clusters. For the equality of means, Welch's t-test or one-way analysis of variance (ANOVA) was performed. The analysis of the contingency table was performed using Fisher's exact test or $\chi^{2}$ test. JMP version 9.0.2 (SAS Institute Japan Co., Ltd., Japan) was used for analysis, and a p-value $<0.05$ (two-tailed) was considered to indicate a statistically significant difference. The multiplicity of the test was not considered, as the analysis of this study was exploratory.

\section{Results}

Patient characteristics. The clinicopathological features of the 1,129 patients are presented in Table I. With regard to tumor location, the percentages obtained were similar for the right- and left-sided colon, and the rectum showed a slightly higher percentage. With regard to the depth of tumor invasion, tumor invasion of mucosa (M), tumor invasion of submucosa (SM) and tumor invasion of muscularis propria (MP) were equivalent to $\leq \mathrm{T} 2$ (Tis, T1 and T2) according to the TNM classification, 7th edition, developed by the Union for International Cancer Control (UICC), and accounted for a low percentage of $17.4 \%$ of the total. Tumor invasion of subserosa (SS), tumor invasion of serosa (SE), direct tumor invasion of other organs or structure in the intestine with serosa (SI), tumor invasion through muscularis propria into non-peritonealized, pericolic, or perirectal tissues (A) and direct tumor invasion of other organs or structure in the intestine without serosa (AI) were equivalent to $\geq \mathrm{T} 3$ (T3, T4a and T4b) according to the TNM classification and accounted for $79.8 \%$. In addition, non-lymph node metastasis (N0) and lymph node metastases ( $\geq \mathrm{N} 1)$ were 41.4 and $56.1 \%$, respectively; stage I/II and stage III/IV disease were 34.0 and $59.6 \%$, respectively, which indicated that the percentage of stage I/II disease tended to be lower.

Intergenic correlation and hierarchical cluster analysis. A summary of the results of the mRNA expression of the 10 target genes is presented in Table II, and the intergenic correlations obtained are listed in Table III. For the patients whose mRNA expression was not detectable (n.d.), the minimum mRNA expression of that gene was inserted as an alternative. The frequency of n.d. was as follows: TYMS, $1(0.1 \%)$; DPYD,

Based on the General Rules for Clinical and Pathological Studies on Cancer of the Colon, Rectum and Anus (Japanese Society for Cancer of the Colon and Rectum, 7th edition) (21). Tumor location: C, cecum; A, ascending colon; $\mathrm{T}$, transverse colon; D, descending colon; S, sigmoid colon. Depth of tumor invasion: M, tumor invasion of mucosa; SM, tumor invasion of submucosa; MP, tumor invasion of muscularis propria; SS, tumor invasion of subserosa SE, tumor invasion of serosa, SI, direct tumor invasion of other organs or structure in the intestine with serosa; A, tumor invasion through muscularis propria into non-peritonealized, pericolic, or perirectal tissues; AI, direct tumor invasion of other organs or structure in the intestine without serosa. 
Table II. Summary of the mRNA expression levels of the 10 genes analyzed in this study in the 1,129 patients.

\begin{tabular}{|c|c|c|c|c|c|}
\hline \multirow[b]{2}{*}{ Gene } & \multicolumn{3}{|c|}{$\begin{array}{l}\text { Gene expression/ } \\
\beta \text {-actin values }\end{array}$} & \multicolumn{2}{|c|}{$\begin{array}{l}\text { Log-transformed } \\
\text { values }\end{array}$} \\
\hline & Median & Min & Max & Mean & SD \\
\hline TYMS & 3.86 & 0.40 & 64.20 & 1.34 & 0.66 \\
\hline$D P Y D$ & 0.28 & 0.03 & 4.61 & -1.30 & 0.85 \\
\hline TYMP & 2.72 & 0.22 & 45.30 & 1.02 & 0.79 \\
\hline DHFR & 4.33 & 0.40 & 27.46 & 1.43 & 0.63 \\
\hline$F P G S$ & 0.59 & 0.15 & 4.49 & -0.53 & 0.49 \\
\hline$G G H$ & 12.71 & 0.26 & 166.47 & 2.45 & 1.09 \\
\hline TOP1 & 2.79 & 0.41 & 107.07 & 1.02 & 0.52 \\
\hline ERCC1 & 1.67 & 0.27 & 18.06 & 0.52 & 0.55 \\
\hline$V E G F$ & 7.39 & 0.93 & 73.19 & 1.99 & 0.65 \\
\hline$E G F R$ & 1.35 & 0.11 & 55.07 & 0.29 & 0.61 \\
\hline
\end{tabular}

35 (3.1\%); TYMP, 9 (0.8\%); DHFR, 1 (0.1\%); FPGS, 2 (0.2\%); GGH, 5 (0.4\%); TOPI, 1 (0.1\%); ERCCl, 2 (0.2\%); VEGF, $1(0.1 \%)$; and $E G F R, 2(0.2 \%)$ (data not shown). The frequency of n.d. was the highest in the $D P Y D$ gene but was in lower than 9 patients $(0.8 \%)$ for the other genes; therefore, it was considered to have little influence on the results of the analysis.

Additionally, the strongest correlation was noted between $D P Y D$ and TYMP $(\mathrm{r}=0.629)$, followed by TYMS and DHFR (r=0.596), GGH and TOPI ( $\mathrm{r}=0.487)$ and FPGS and $E R C C 1(\mathrm{r}=0.456)$. In a hierarchical cluster analysis of mRNA expression, the data were roughly classified into 2 groups: cluster A, in which the mRNA expression of all 10 genes was significantly low, and cluster B, in which the mRNA expression was significantly higher (Fig. 1 and Table IV). The associations between the 2 clusters and the clinicopathological features are summarized in Table V. A significant difference was noted between the histology, lymphatic invasion, lymph node metastasis and stage. There was a tendency for low-frequency mucinous adenocarcinoma in cluster B and the frequencies of non-lymphatic invasion (ly0), N0 and stage I/II disease were also higher in cluster $\mathrm{B}$.

Association of mRNA expression and clinicopathological features. The association between the mRNA expression of 10 target genes and the clinicopathological features is presented in Table VI. In relation to the location of the tumor, a significant difference was noted in all genes apart from $V E G F$ when 4 locations were compared (the right- and left-sided colon, rectosigmoid and rectum), with the exception of the proctos; only 4 patients had a tumor at this location. Specifically, $T Y M S$ and $D P Y D$ showed the highest level of expression in the right-sided colon; $G G H$ and $E G F R$ showed the highest level of expression in the left-sided colon; DHFR, FPGS, TOPI and $E R C C 1$ showed the highest level of expression in the rectosigmoid; TYMP showed approximately equivalent expression levels in the right-sided colon and rectum, where the expression was higher than in the other locations. On comparing two locations (right- and left-sided colon cancer) in patients with colon cancer only, a significantly high level of expression was noted in right-sided colon cancer for TYMS (fold change, 1.22; $\mathrm{p}=0.0001), D P Y D$ (1.27-fold; $\mathrm{p}=0.001)$, TYMP (1.31fold; $\mathrm{p}<0.0001)$, and $F P G S$ (1.09-fold; $\mathrm{p}=0.020)$. By contrast, a significantly high level of expression was observed in leftsided colon cancer for the GGH (1.74-fold; p $<0.0001), T O P 1$ (1.17-fold; $\mathrm{p}<0.0001)$ and EGFR (1.15-fold; $\mathrm{p}=0.006)$ genes, and no significant difference was noted for DHFR (1.06-fold; $\mathrm{p}=0.226), E R C C l$ (1.08-fold; $\mathrm{p}=0.084)$ and VEGF (1.07-fold; $\mathrm{p}=0.191$ ) (data not shown). For histology, there was a significant difference in 7 genes, excluding $D H F R, V E G F$ and $E G F R$. For $T Y M S, D P Y D$ and TYMP, the highest levels of expression were observed in patients with poorly differentiated adenocarcinoma, while the lowest levels of expression were observed in patients with well/moderately differentiated adenocarcinoma. For $F P G S, G G H, T O P 1$ and $E R C C 1$, the lowest levels of expression were observed in patients with mucinous adenocarcinoma; for $F P G S$ and ERCC1, the highest level of expression was observed in patients with poorly differentiated adenocarcinoma; and for $G G H$ and $T O P 1$, the highest level of expression was observed in patients with well/moderately differentiated adenocarcinoma. As regards the depth of tumor invasion, a significantly higher

Table III. Correlations of the mRNA expression of the 10 log-transformed genes.

\begin{tabular}{|c|c|c|c|c|c|c|c|c|c|c|}
\hline Gene & TYMS & $D P Y D$ & $T Y M P$ & $D H F R$ & $F P G S$ & $G G H$ & TOP1 & ERCCl & $V E G F$ & $E G F R$ \\
\hline$T Y M S$ & 1 & & & 0.596 & & & & 0.427 & & \\
\hline$D P Y D$ & & 1 & 0.629 & & & & & & & \\
\hline$T Y M P$ & & 0.629 & 1 & & & & & & & \\
\hline DHFR & 0.596 & & & 1 & & 0.438 & 0.387 & 0.373 & & \\
\hline$F P G S$ & & & & & 1 & & & 0.456 & & \\
\hline$G G H$ & & & & 0.438 & & 1 & 0.487 & & & \\
\hline TOP1 & & & & 0.387 & & 0.487 & 1 & & 0.341 & \\
\hline ERCCl & 0.427 & & & 0.373 & 0.456 & & & 1 & & \\
\hline$V E G F$ & & & & & & & 0.341 & & 1 & \\
\hline$E G F R$ & & & & & & & & & & 1 \\
\hline
\end{tabular}

Only Pearson's correlations $>0.3$ are shown $(\mathrm{p}<0.0001)$. 


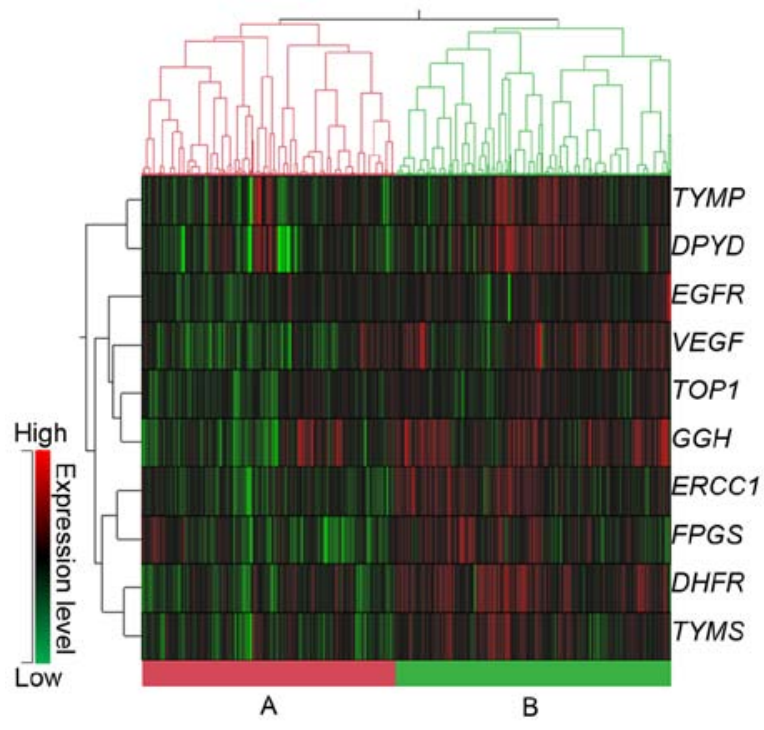

Figure 1. Hierarchical clustering of 10 gene mRNA expression.

Table IV. Log-transformed mRNA expression levels of two clusters.

\begin{tabular}{lccc}
\hline & \multicolumn{2}{c}{ Gene expression/ $\beta$-actin values } & \\
\cline { 2 - 3 } Gene & Cluster A $(\mathrm{n}=539)$ & Cluster B $(\mathrm{n}=590)$ & p-value \\
\hline TYMS & $1.04 \pm 0.61$ & $1.61 \pm 0.60$ & $<0.0001$ \\
DPYD & $-1.62 \pm 0.88$ & $-1.00 \pm 0.72$ & $<0.0001$ \\
TYMP & $0.82 \pm 0.81$ & $1.20 \pm 0.72$ & $<0.0001$ \\
DHFR & $1.08 \pm 0.56$ & $1.75 \pm 0.51$ & $<0.0001$ \\
FPGS & $-0.71 \pm 0.46$ & $-0.37 \pm 0.46$ & $<0.0001$ \\
GGH & $1.97 \pm 1.11$ & $2.90 \pm 0.86$ & $<0.0001$ \\
TOP1 & $0.84 \pm 0.48$ & $1.19 \pm 0.49$ & $<0.0001$ \\
ERCC1 & $0.24 \pm 0.44$ & $0.78 \pm 0.52$ & $<0.0001$ \\
VEGF & $1.69 \pm 0.59$ & $2.26 \pm 0.59$ & $<0.0001$ \\
EGFR & $0.11 \pm 0.54$ & $0.45 \pm 0.64$ & $<0.0001$ \\
\hline
\end{tabular}

p-values were calculated by Welch's t-test.

level of expression was found in $\mathrm{T} 1 / 2$ as compared to $\mathrm{T} 3 / 4$ for TYMS, TYMP, DHFR, and ERCCl, while higher expression was found in T3/4 for $V E G F$ only. For lymph node metastasis, there was a significant difference in TYMS, DHFR and ERCCI; a significantly higher level of expression was found in N0 than $\mathrm{N} \geq 1$. For stage, there was a significant difference in $T Y M S$, $T Y M P, D H F R$ and $E R C C 1$, whereas a significantly higher level of expression was found in stage I/II than stage III/IV disease.

Comparison of clinicopathological features in right- or leftsided colon cancer. A comparison of the clinicopathological features between the patients with right- and left-sided colon cancer is presented in Table VII. A significant difference was found with respect to age, histology, lymph node metastasis and stage. The frequencies of elderly individuals, poorly differentiated and mucinous adenocarcinomas, N0 and stage I/II disease tended to be higher in right-sided colon cancer than left-sided
Table V. Comparison of clinicopathological features in two clusters of gene expression.

\begin{tabular}{|c|c|c|c|}
\hline \multirow[b]{2}{*}{ Clinicopathological features } & \multicolumn{2}{|c|}{ No. of patients } & \multirow[b]{2}{*}{$\mathrm{p}$-value } \\
\hline & Cluster A & Cluster B & \\
\hline \multicolumn{4}{|l|}{ Age (years) } \\
\hline$<67$ & 252 & 273 & 0.394 \\
\hline$\geq 67$ & 253 & 305 & \\
\hline \multicolumn{4}{|l|}{ Gender } \\
\hline Male & 312 & 350 & 0.761 \\
\hline Female & 221 & 238 & \\
\hline \multicolumn{4}{|l|}{ Tumor location } \\
\hline Right-sided colon & 150 & 174 & 0.305 \\
\hline Left-sided colon & 156 & 161 & \\
\hline Rectosigmoid & 47 & 70 & \\
\hline Rectum & 177 & 181 & \\
\hline \multicolumn{4}{|l|}{ Histology } \\
\hline Well/moderately differentiated & 479 & 525 & 0.008 \\
\hline Poorly differentiated & 15 & 20 & \\
\hline Mucinous & 24 & 8 & \\
\hline \multicolumn{4}{|l|}{ Lymphatic invasion } \\
\hline ly0 & 129 & 163 & 0.039 \\
\hline ly1, ly2, ly3 & 383 & 363 & \\
\hline \multicolumn{4}{|l|}{ Venous invasion } \\
\hline v0 & 171 & 193 & 0.241 \\
\hline $\mathrm{v} 1, \mathrm{v} 2, \mathrm{v} 3$ & 341 & 329 & \\
\hline \multicolumn{4}{|l|}{ Depth of tumor invasion } \\
\hline $\mathrm{T} 1, \mathrm{~T} 2$ & 102 & 95 & 0.238 \\
\hline $\mathrm{T} 3, \mathrm{~T} 4$ & 424 & 477 & \\
\hline \multicolumn{4}{|l|}{ Lymph node metastasis } \\
\hline No & 202 & 265 & 0.006 \\
\hline $\mathrm{N} 1, \mathrm{~N} 2, \mathrm{~N} 3$ & 327 & 306 & \\
\hline \multicolumn{4}{|l|}{ Stage } \\
\hline I, II & 166 & 218 & 0.013 \\
\hline III, IV & 345 & 328 & \\
\hline
\end{tabular}

p-values were calculated by Fisher's exact test or $\chi^{2}$ test.

colon cancer. The frequency of female patients tended to be higher for right- than left-sided colon cancer although the difference was not significant.

\section{Discussion}

In this study, FFPE tumor specimens of primary tumors obtained from 1,129 patients with colorectal cancer were used. We measured mRNA expression levels of 10 genes associated with the effects of standard chemotherapy for colorectal cancer (TYMS, DPYD, TYMP, DHFR, FPGS, GGH, TOPI, $E R C C 1, V E G F$ and $E G F R$ ), and determined their association with clinicopathological features, in particular, the location of colorectal cancer. 


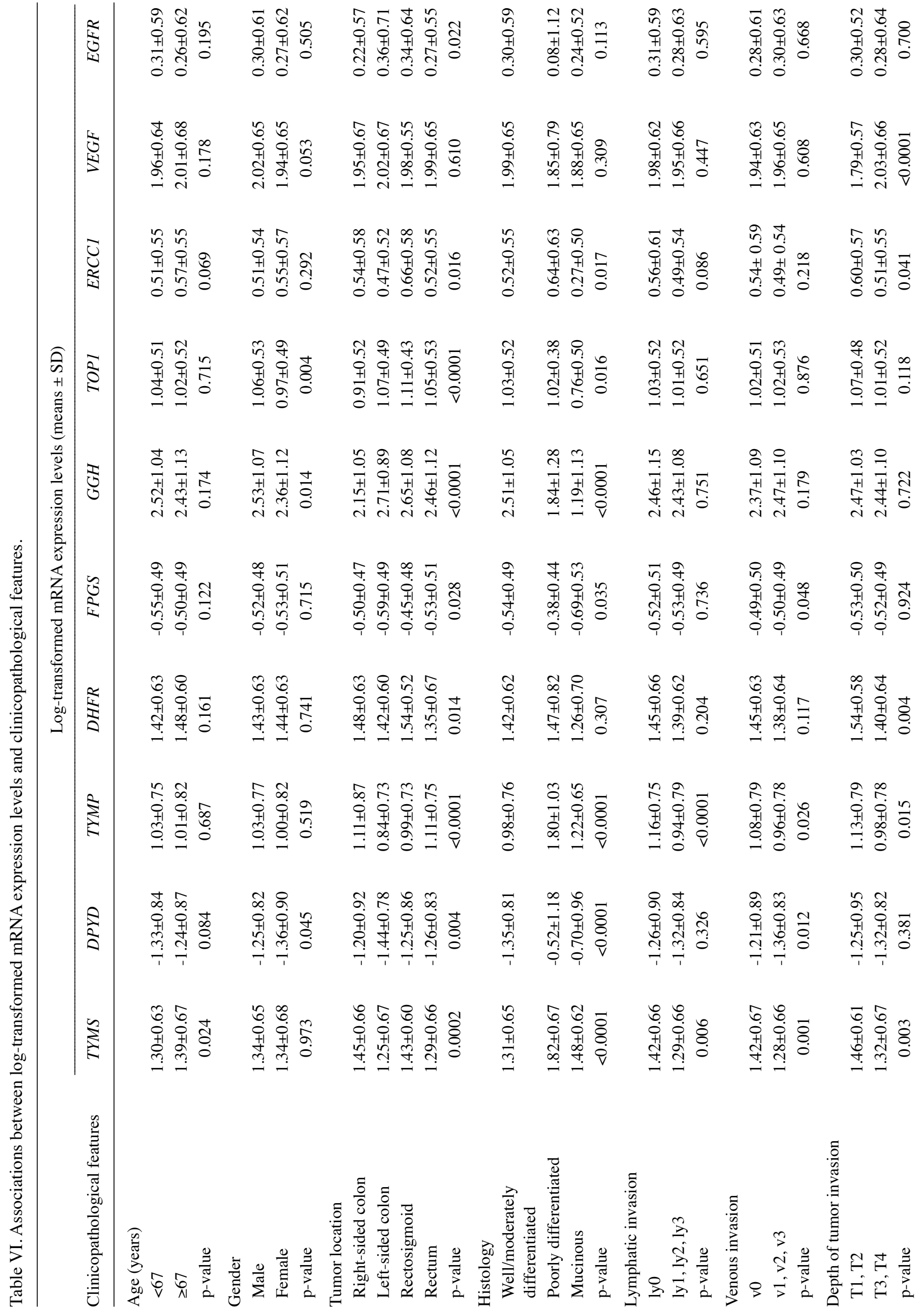


Table VII. Comparison of clinicopathological features with the location of the colorectal cancer.

\begin{tabular}{|c|c|c|c|}
\hline \multirow[b]{2}{*}{$\begin{array}{l}\text { Clinicopathological } \\
\text { features }\end{array}$} & \multicolumn{2}{|c|}{ No. of patients } & \multirow[b]{2}{*}{ p-value } \\
\hline & $\begin{array}{l}\text { Right-sided } \\
\text { colon }\end{array}$ & $\begin{array}{l}\text { Left-sided } \\
\text { colon }\end{array}$ & \\
\hline \multicolumn{4}{|l|}{ Age (years) } \\
\hline$<67$ & 105 & 159 & $<0.0001$ \\
\hline$\geq 67$ & 205 & 148 & \\
\hline \multicolumn{4}{|l|}{ Gender } \\
\hline Male & 166 & 183 & 0.175 \\
\hline Female & 151 & 133 & \\
\hline \multicolumn{4}{|l|}{ Histology } \\
\hline $\begin{array}{l}\text { Well/moderately } \\
\text { differentiated }\end{array}$ & 279 & 291 & 0.001 \\
\hline Poorly differentiated & 16 & 9 & \\
\hline Mucinous & 17 & 2 & \\
\hline \multicolumn{4}{|l|}{ Lymphatic invasion } \\
\hline ly0 & 88 & 73 & 0.197 \\
\hline ly1, ly2, ly3 & 211 & 224 & \\
\hline \multicolumn{4}{|l|}{ Venous invasion } \\
\hline v0 & 115 & 96 & 0.145 \\
\hline $\mathrm{v} 1, \mathrm{v} 2, \mathrm{v} 3$ & 184 & 199 & \\
\hline \multicolumn{4}{|l|}{ Depth of tumor invasion } \\
\hline $\mathrm{T} 1, \mathrm{~T} 2$ & 40 & 32 & 0.382 \\
\hline $\mathrm{T} 3, \mathrm{~T} 4$ & 278 & 282 & \\
\hline \multicolumn{4}{|l|}{ Lymph node metastasis } \\
\hline NO & 146 & 115 & 0.029 \\
\hline $\mathrm{N} 1, \mathrm{~N} 2, \mathrm{~N} 3$ & 173 & 196 & \\
\hline \multicolumn{4}{|l|}{ Stage } \\
\hline I, II & 118 & 93 & 0.041 \\
\hline III, IV & 186 & 210 & \\
\hline
\end{tabular}

p-values were calculated by Fisher's exact test or $\chi^{2}$ test.

From the results of mRNA expression analysis of the 10 genes included in this study, the strongest correlation was noted between $D P Y D$ and TYMP. Several studies have reported a similar correlation between expression levels in colorectal cancer (26-33). DPYD and TYMP encode enzymes related to the metabolism of 5-FU, and it seems likely that the expression levels of these genes in colorectal cancer tissues are co-regulated. In addition, the results from this study indicate a weaker correlation between TYMS and DHFR than that between DPYD and TYMP. Uetake et al also reported a similar correlation in stage III colon cancer (33). Considering that DHFR plays an important role in the generation of 5,10-methylene-THF as a co-factor of the TYMS response, it is plausible that the level of gene expression of both enzymes is correlated.

It has previously been reported that colorectal cancer, particularly colon cancer, exhibits embryologic, morphologic, physiologic and molecular differences between the right and left sides, which leads to differences in the prognosis of 
patients and their sensitivity to chemotherapy (15-18). In relation to the clinicopathological features, in these studies, it has been posited that the numbers of elderly individuals, women, mucinous adenocarcinoma, high grade (poorly differentiated/ undifferentiated) and negative lymph node metastasis is higher in right- than left-sided colon cancer. In our study, similar, reproducible results were noted. As regards the molecular features, a microsatellite instability phenotype $\left(\mathrm{MSI}^{+}\right)$and $\mathrm{CpG}$ island methylator phenotype $\left(\mathrm{CIMP}^{+}\right)$are common in right-sided colon cancer, whereas chromosomal instability is common in left-sided colon cancer (15-18). Kawakami et al (34) analyzed the association between the $\mathrm{CIMP}^{+}$status and the expression level of gene-related folate and nucleotide metabolism in the primary tumors of 114 patients from Australia with colorectal cancer. They reported that the low-level expression of $G G H$ and high-level expression of TYMP were very strongly associated with $\mathrm{CIMP}^{+}$and $\mathrm{CIMP}^{+}$-related clinicopathological and molecular features. They also reported that a significantly higher level of TYMP expression and a significantly lower level of $G G H$ expression were found in right- as compared to left-sided colorectal cancer (34). Moreover, they pointed out the possibility that low-level $G G H$ expression may lead to the maintenance of folate in the polyglutamate form in the cells, which is associated with DNA hypermethylation, and that 5-FU plus leucovorin therapy may then be more effective (34). In a study of 76 Japanese patients with stage II/III colorectal cancer, Sadahiro et al reported that a significantly high level of TYMP expression was found in cases of right rather than left-sided colorectal cancer and that preoperative uracil-tegafur (UFT) plus leucovorin therapy resulted in a significantly strong histological response (35). In the present study, in relation to right-sided colon cancer, we noted a significantly higher level of TYMP expression and a lower level $G G H$ expression than in left-sided colon cancer. Considering these results, it may be worthwhile to determine the physiological significance of TYMP and GGH expression in patients with colorectal cancer in the future, in order to examine the hypothesis that 5-FU-based chemotherapy combined with leucovorin may be more effective in cases of right-sided colon cancer.

Ricciardiello et al reported that TYMS protein expression, as assessed by immunohistochemistry (IHC), did not differ significantly between right- and left-sided colorectal cancer, but a significantly high level of expression in $\mathrm{MSI}^{+}$colorectal cancer was noted (36). Jensen et al also reported that the protein expression of TYMS (studied by IHC) was significantly high in $\mathrm{MSI}^{+}$colorectal cancer (37). On the other hand, Sinicrope et al reported that there was no correlation between the $\mathrm{MSI}^{+}$status and TYMS protein expression (as measured by IHC) (38). Thus, the association between TYMS protein expression and the $\mathrm{MSI}^{+}$status remains controversial. In our study, as regards TYMS, its mRNA expression was found to be higher in right-sided colon cancer in which $\mathrm{MSI}^{+}$was common, than in left-sided colon cancer. The analysis of the clinicopathological features of all the patients with colorectal cancer in this study revealed a high level of TYMS expression in the elderly, those with poorly differentiated adenocarcinoma and N0, which are common in right-sided colon cancer. However, as regards the association between TYMS expression and the location of colorectal cancer, further studies, which will include the association of TYMS with molecular features, such as the $\mathrm{MSI}^{+}$ status, are warranted in the future.

Recently, Missiaglia et al performed gene expression analysis of a colon cancer dataset on 914 patients who were registered in The Cancer Genome Atlas (TCGA) collection and Pan European Trial Adjuvant Colon Cancer (PETACC3) adjuvant chemotherapy trial. According to their study, the expression of EGFR and human epidermal growth factor receptor 2 (HER2) was advanced in left- rather than right- sided colon cancer, which activated EGF signaling, and the usefulness of anti-EGFR agents was suggested (39). Similarly, the results of our study demonstrated that a significantly higher level of $E G F R$ expression was noted in left- rather than right-sided colon cancer. Hence, it is necessary to perform research on individualized treatment which combines the location of colorectal cancer and $E G F R$ expression levels with the mutation status of KRAS and $B R A F$, as well as $E G F R$ inhibitors.

Loupakis et al analyzed three independent cohorts in order to examine the association between the primary tumor of metastatic colorectal cancer and the efficacy of first-line chemotherapy. They reported that patients with primary tumors on the left side of the colon achieved significantly superior overall survival than those with primary tumors on the right side (40). They also analyzed gene expression in an exploratory manner using the archival series of 181 independent primary tumor samples. They inferred that the mRNA expression of ERCC1 was significantly higher in right- rather than left-sided colorectal cancer, although the mRNA expression level of $V E G F$ ligands $A, B$ and $C$, as well as VEGF receptors 1 and 2 showed no difference between right- and left-sided colorectal cancer. This is, perhaps, one of the factors involved in chemoresistance in patients with right-sided colorectal cancer (40). In our study, although $V E G F$ exhibited few differences in the level of expression according to the location of the tumor and the expression of ERCCI was the lowest in the patients with leftsided colon cancer, the level of expression for ERCCl was the highest in the rectosigmoid. Additionally, for $E R C C 1$, there was no significant difference in the comparison between right- and left-sided colon cancer. Further research on the differences in sensitivity to oxaliplatin between patients with right- and left-sided colorectal cancer and the association with ERCCI expression is warranted.

In our analysis of the association between gene expression and five factors related to the extent of cancer, namely lymphatic invasion, vein invasion, depth of tumor invasion, lymph node metastasis and stage, a significant difference was noted in all the five factors for TYMS; whereas DPYD, TYMP, DHFR, FPGS, ERCCI and VEGF showed a significant difference in 1-4 factors. Of these, with the exception that the $V E G F$ gene showed a significantly higher level of expression in T3/4 than $\mathrm{T} 1 / 2$, high-level expression was found in the group at a less-advanced stage rather than the group in the more advanced stages of cancer. This tendency is consistent with the results of the hierarchical cluster analysis. However, a comparison of patient characteristics was made between the 1,129 Japanese patients with colorectal cancer in our study and the data from 30,002 patients who were registered with the Japanese Society for Cancer of the Colon and Rectum between 1993 and 1997 (41), as well as the data from 621 patients who were registered in a single institution in Japan between 1992 and 2002 (42). This 
comparison revealed that, although age, gender, tumor location and histology had generally similar distributions, the percentages of T1/2, N0 and stage I/II were clearly low, and as a whole, the stage of cancer tended to be more advanced in our study. Consequently, it is likely necessary to consider the data on the association between gene expression levels in this study and the clinicopathological features associated with the progress of cancer with caution.

In this large-scale population study, we clarified the association between the mRNA expression of genes associated with the effects of standard chemotherapy for colorectal cancer and clinicopathological features, particularly the location of the colorectal cancer. It is hoped that the results of this study will become useful reference data for conducting research on individualized chemotherapy for colorectal cancer that takes into consideration the location of colorectal cancer.

\section{Acknowledgements}

The authors wish to thank all of the patients who participated in this study, and the clinicians at the hospitals named in the Appendix, who collaborated with us in measuring gene expression in the colorectal cancer specimens. In addition, we would like to thank Editage (www.editage.jp) for English language editing.

\section{References}

1. Ferlay J, Soerjomataram I, Dikshit R, Eser S, Mathers C, Rebelo M, Parkin DM, Forman D and Bray F: Cancer incidence and mortality worldwide: sources, methods and major patterns in GLOBOCAN 2012. Int J Cancer 136: E359-E386, 2015.

2. Meyerhardt JA and Mayer RJ: Systemic therapy for colorectal cancer. N Engl J Med 352: 476-487, 2005.

3. Wilson PM, Danenberg PV, Johnston PG, Lenz HJ and Ladner RD: Standing the test of time: targeting thymidylate biosynthesis in cancer therapy. Nat Rev Clin Oncol 11: 282-298, 2014.

4. van Kuilenburg AB: Dihydropyrimidine dehydrogenase and the efficacy and toxicity of 5-fluorouracil. Eur J Cancer 40: 939-950, 2004.

5. Toi M, Atiqur Rahman M, Bando H and Chow LW: Thymidine phosphorylase (platelet-derived endothelial-cell growth factor) in cancer biology and treatment. Lancet Oncol 6: 158-166, 2005.

6. Salonga D, Danenberg KD, Johnson M, Metzger R, Groshen S, Tsao-Wei DD, Lenz HJ, Leichman CG, Leichman L, Diasio RB and Danenberg PV: Colorectal tumors responding to 5-fluorouracil have low gene expression levels of dihydropyrimidine dehydrogenase, thymidylate synthase, and thymidine phosphorylase. Clin Cancer Res 6: 1322-1327, 2000.

7. Soong R, Shah N, Salto-Tellez M, Tai BC, Soo RA, Han HC, Ng SS, Tan WL, Zeps N, Joseph D, et al: Prognostic significance of thymidylate synthase, dihydropyrimidine dehydrogenase and thymidine phosphorylase protein expression in colorectal cancer patients treated with or without 5-fluorouracil-based chemotherapy. Ann Oncol 19: 915-919, 2008.

8. Laufman LR, Krzeczowski KA, Roach R and Segal M: Leucovorin plus 5-fluorouracil: an effective treatment for metastatic colon cancer. J Clin Oncol 5: 1394-1400, 1987.

9. Sakamoto E, Tsukioka S, Oie S, Kobunai T, Tsujimoto H, Sakamoto K, Okayama Y, Sugimoto Y, Oka T, Fukushima M and Oka T: Folylpolyglutamate synthase and gamma-glutamyl hydrolase regulate leucovorin-enhanced 5-fluorouracil anticancer activity. Biochem Biophys Res Commun 365: 801-807, 2008.

10. Pommier Y: Topoisomerase I inhibitors: camptothecins and beyond. Nat Rev Cancer 6: 789-802, 2006.

11. Shirota Y, Stoehlmacher J, Brabender J, Xiong YP, Uetake H, Danenberg KD, Groshen S, Tsao-Wei DD, Danenberg PV and Lenz HJ: ERCC1 and thymidylate synthase mRNA levels predict survival for colorectal cancer patients receiving combination oxaliplatin and fluorouracil chemotherapy. J Clin Oncol 19: 4298-4304, 2001.
12. Bohanes P, Labonte MJ and Lenz HJ: A review of excision repair cross-complementation group 1 in colorectal cancer. Clin Colorectal Cancer 10: 157-164, 2011.

13. Hicklin DJ and Ellis LM: Role of the vascular endothelial growth factor pathway in tumor growth and angiogenesis. J Clin Oncol 23: 1011-1027, 2005.

14. Baselga $J$ and Arteaga CL: Critical update and emerging trends in epidermal growth factor receptor targeting in cancer. J Clin Oncol 23: 2445-2459, 2005.

15. Elsaleh H, Joseph D, Grieu F, Zeps N, Spry N and Iacopetta B: Association of tumour site and sex with survival benefit from adjuvant chemotherapy in colorectal cancer. Lancet 355: 1745-1750, 2000.

16. Iacopetta B: Are there two sides to colorectal cancer? Int J Cancer 101: 403-408, 2002.

17. Gervaz P, Bucher P and Morel P: Two colons-two cancers: paradigm shift and clinical implications. J Surg Oncol 88: 261-266, 2004.

18. Söreide K, Janssen EA, Söiland H, Körner H and Baak JP: Microsatellite instability in colorectal cancer. Br J Surg 93: 395-406, 2006

19. Fukushima M, Morita M, Ikeda K and Nagayama S: Population study of expression of thymidylate synthase and dihydropyrimidine dehydrogenase in patients with solid tumors. Int J Mol Med 12: 839-844, 2003.

20. Fukui Y, Oka T, Nagayama S, Danenberg PV, Danenberg KD and Fukushima M: Thymidylate synthase, dihydropyrimidine dehydrogenase, orotate phosphoribosyltransferase mRNA and protein expression levels in solid tumors in large scale population analysis. Int J Mol Med 22: 709-716, 2008.

21. General Rules for Clinical and Pathological Studies on Cancer of the Colon, Rectum and Anus. Japanese Society for Cancer of the Colon and Rectum (eds). 7th edition, Kanehara \& Co., Ltd., Kanehara, Tokyo, 2006.

22. Lord RV, Salonga D, Danenberg KD, Peters JH, DeMeester TR, Park JM, Johansson J, Skinner KA, Chandrasoma P, DeMeester SR, et al: Telomerase reverse transcriptase expression is increased early in the Barrett's metaplasia, dysplasia, adenocarcinoma sequence. J Gastrointest Surg 4: 135-142, 2000.

23. Heid CA, Stevens J, Livak KJ and Williams PM: Real time quantitative PCR. Genome Res 6: 986-994, 1996.

24. Gibson UE, Heid CA and Williams PM: A novel method for real time quantitative RT-PCR. Genome Res 6: 995-1001, 1996.

25. Kuramochi H, Hayashi K, Uchida K, Miyakura S, Shimizu D, Vallböhmer D, Park S, Danenberg KD, Takasaki K and Danenberg PV: Vascular endothelial growth factor messenger RNA expression level is preserved in liver metastases compared with corresponding primary colorectal cancer. Clin Cancer Res 12: 29-33, 2006.

26. Ichikawa W, Uetake H, Shirota Y, Yamada H, Takahashi T, Nihei Z, Sugihara K, Sasaki Y and Hirayama R: Both gene expression for orotate phosphoribosyltransferase and its ratio to dihydropyrimidine dehydrogenase influence outcome following fluoropyrimidine-based chemotherapy for metastatic colorectal cancer. Br J Cancer 89: 1486-1492, 2003.

27. Yoshinare K, Kubota T, Watanabe M, Wada N, Nishibori H, Hasegawa H, Kitajima M, Takechi T and Fukushima M: Gene expression in colorectal cancer and in vitro chemosensitivity to 5-fluorouracil: a study of 88 surgical specimens. Cancer Sci 94: 633-638, 2003.

28. Inokuchi M, Uetake H, Shirota Y, Yamada H, Tajima M and Sugihara K: Gene expression of 5-fluorouracil metabolic enzymes in primary colorectal cancer and corresponding liver metastasis. Cancer Chemother Pharmacol 53: 391-396, 2004.

29. Uchida K, Danenberg PV, Danenberg KD and Grem JL: Thymidylate synthase, dihydropyrimidine dehydrogenase, ERCC1, and thymidine phosphorylase gene expression in primary and metastatic gastrointestinal adenocarcinoma tissue in patients treated on a phase I trial of oxaliplatin and capecitabine. BMC Cancer 8: 386, 2008.

30. Sameshima S, Tomozawa S, Kojima M, Koketsu S, Motegi K, HorikoshiH,OkadaT,Kon Y and Sawada T:5-Fluorouracil-related gene expression in primary sites and hepatic metastases of colorectal carcinomas. Anticancer Res 28 (3A): 1477-1481, 2008.

31. Kumamoto K, Kuwabara K, Tajima Y, Amano K, Hatano S, Ohsawa T, Okada N, Ishibashi K, Haga N and Ishida $\mathrm{H}$ : Thymidylate synthase and thymidine phosphorylase mRNA expression in primary lesions using laser capture microdissection is useful for prediction of the efficacy of FOLFOX treatment in colorectal cancer patients with liver metastasis. Oncol Lett 3: 983-989, 2012. 
32. Goto T, Shinmura K, Yokomizo K, Sakuraba K, Kitamura Y, Shirahata A, Saito M, Kigawa G, Nemoto H, Sanada Y and Hibi K: Expression levels of thymidylate synthase, dihydropyrimidine dehydrogenase, and thymidine phosphorylase in patients with colorectal cancer. Anticancer Res 32: 1757-1762, 2012.

33. Uetake H, Ishikawa T, Matsui S and Sugihara K: Gene expression analysis as a biomarker study for ACTS-CC trial (TRICC0706), a phase III trial for adjuvant chemotherapy of S-1 and UFT/LV against stage III colon cancer in Japan. Cancer Res 72 (8 Suppl): $4516,2012$.

34. Kawakami K, Ooyama A, Ruszkiewicz A, Jin M, Watanabe G, Moore J, Oka T, Iacopetta B and Minamoto T: Low expression of gamma-glutamyl hydrolase mRNA in primary colorectal cancer with the $\mathrm{CpG}$ island methylator phenotype. Br J Cancer 98: 1555-1561, 2008.

35. Sadahiro S, Suzuki T, Tanaka A, Okada K, Nagase H and Uchida J: Association of right-sided tumors with high thymidine phosphorylase gene expression levels and the response to oral uracil and tegafur/leucovorin chemotherapy among patients with colorectal cancer. Cancer Chemother Pharmacol 70: 285-291, 2012.

36. Ricciardiello L, Ceccarelli C, Angiolini G, Pariali M, Chieco P, Paterini P, Biasco G, Martinelli GN, Roda E and Bazzoli F: High thymidylate synthase expression in colorectal cancer with microsatellite instability: Implications for chemotherapeutic strategies. Clin Cancer Res 11: 4234-4240, 2005.

37. Jensen SA, Vainer B,Kruhøffer M and Sørensen JB: Microsatellite instability in colorectal cancer and association with thymidylate synthase and dihydropyrimidine dehydrogenase expression. BMC Cancer 9: 25, 2009.

38. Sinicrope FA, Rego RL, Halling KC, Foster NR, Sargent DJ, La Plant B, French AJ, Allegra CJ, Laurie JA, Goldberg RM, et al: Thymidylate synthase expression in colon carcinomas with microsatellite instability. Clin Cancer Res 12: 2738-2744, 2006.

39. Missiaglia E, Jacobs B, D'Ario G, Di Narzo AF, Soneson C, Budinska E, Popovici V, Vecchione L, Gerster S, Yan P, et al: Distal and proximal colon cancers differ in terms of molecular, pathological, and clinical features. Ann Oncol 25: 1995-2001, 2014.

40. Loupakis F, Yang D, Yau L, Feng S, Cremolini C, Zhang W, Maus MK, Antoniotti C, Langer C, Scherer SJ, et al: Primary tumor location as a prognostic factor in metastatic colorectal cancer. J Natl Cancer Inst 107: dju427, 2015.
41. Kotake K, Honjo S, Sugihara K, Kato T, Kodaira S, Takahashi T, Yasutomi M, Muto T and Koyama Y: Changes in colorectal cancer during a 20 -year period: an extended report from the multi-institutional registry of large bowel cancer, Japan. Dis Colon Rectum 46 (Suppl): S32-S43, 2003.

42. Sakamoto K, Machi J, Prygrocki M, Watanabe T, Hosoda S, Sugano M, Tomiki $\mathrm{Y}$ and Kamano T: Comparison of characteristics and survival of colorectal cancer between Japanese-Americans in Hawaii and native Japanese in Japan. Dis Colon Rectum 49: 50-57, 2006.

\section{Appendix}

Collaborating hospitals: Chiba University Hospital, Fujita Health University Hospital, Gifu University Hospital, Hachioji Digestive Disease Hospital, Handa City Hospital, Himeji Medical Center, Hyogo Prefectural Kakogawa Medical Center, JCHO Kobe Central Hospital, Kanto Rosai Hospital, Keio University Hospital, Kinki University Hospital, Kitano Hospital, Kobe City Medical Center General Hospital, Kobe City Medical Center West Hospital, Kohsei Chuo General Hospital, Nagoya University Hospital, National Center for Global Health and Medicine Center Hospital, Nihon University Itabashi Hospital, Niitsu Medical Center Hospital, Omori Red Cross Hospital, Osaka Rosai Hospital, Osaka University Hospital, Saitama Medical Center, Sakai City Hospital, Sendai Red Cross Hospital, Showa University Fujigaoka Hospital, Showa University Hospital, The Jikei University Hospital, Tohoku Rosai Hospital, Tohoku University Hospital, Toho University Omori Medical Center, Tokushima Red Cross Hospital, Tokyo Medical University Hachioji Medical Center, Tokyo Metropolitan Cancer and Infectious Diseases Center Komagome Hospital, Tokyo Metropolitan Tama Medical Center, Totsuka Kyoritsu Second Hospital, Toyohashi Municipal Hospital, University of Yamanashi Hospital, Yamaguchi University Hospital. 